\title{
Perdas produtivas e financeiras geradas por vibrações e impactos em esteiras transportadoras em aviários: revisão bibliográfica
}

\author{
Productive and financial losses generated by vibrations and impacts on conveyor belts in poultry
}

houses: bibliographic review

Pérdidas productivas y económicas generadas por vibraciones e impactos en cintas transportadoras en aviarios: revisión bibliográfica

\author{
Mario Mollo Neto \\ ORCID: https://orcid.org/0000-0002-8341-4190 \\ Universidade Estadual Paulista, Brasil \\ E-mail: mario.mollo@unesp.br
}

\begin{abstract}
Resumo
O Brasil é um grande produtor de ovos e o Estado de São Paulo é que mais produz ovos do Brasil, sendo a região de Tupã a maior produtora no Estado com 55\% da produção e, em 2018 Bastos, a cidade com maior produção, aportou $36 \%$ do total paulista. Assim, com estes volumes de produção, é possível compreender a importância deste setor produtivo, que demanda inovações tecnológicas para garantir a permanência no mercado e a eficiência na produção. A implantação de novas tecnologias para automatização da produção de ovos, nem sempre trazem só resultados positivos, mas demandam ajustes para que se evitem perdas durante o processo produtivo. Neste contexto, destaca-se o problema advindo dos sistemas automatizados de transporte dos ovos, que podem causar perdas produtivas e financeiras para as granjas. Há perdas na linha de produção durante o transporte automatizado desde os galpões até o processo final de expedição, onde vibrações e impactos causam danos, assim como os tempos de exposição ao sol. Considerando o cenário descrito, neste artigo o gol baseia-se na prospecção da bibliografia que demonstrou que já há tecnologia desenvolvida pelos pares para dar suporte integral ao futuro desenvolvimento tecnológico de um sistema eletrônico autônomo para ser lançado ao sistema de transporte, coletando dados dos obstáculos, sua posição e condições de exposição às intempéries, passível de comunicação com computadores do sistema de gestão dos aviários e que possam dar informações de forma a mitigar as perdas produtivas, ampliando a competitividade e segurança alimentar das granjas.
\end{abstract}

Palavras-chave: Revisão; Coletor de dados; Software; Produção de ovos; Vibração.

\begin{abstract}
Brazil is a major producer of eggs and the State of São Paulo is the one that produces the most eggs in Brazil, with the Tupã region being the largest producer in the State with $55 \%$ of production and, in 2018 Bastos, the city with the largest production, contributed $36 \%$ of the São Paulo total. Thus, with these production volumes, it is possible to understand the importance of this productive sector, which demands technological innovations to guarantee permanence in the market and efficiency in production. The implementation of new technologies to automate the production of eggs does not always bring only positive results but requires adjustments to avoid losses during the production process. In this context, the problem arising from the automated egg transport systems, which can cause productive and financial losses for the farms, stands out. There are losses in the production line during automated transport from the warehouses to the final shipping process, where vibrations and impacts cause damage, as well as the times of exposure to the sun. Considering the scenario described, in this article the goal is based on prospecting the bibliography that demonstrated that there is already technology developed by peers to fully support the future technological development of an autonomous electronic system to be launched to the transport system, collecting data on obstacles, its position, and conditions of exposure to bad weather, which can be communicated with computers in the aviary management system and which can provide information in order to mitigate production losses, increasing the competitiveness and food security of the farms.
\end{abstract}

Keywords: Review; Data collector; Software; Egg production; Vibration.

\section{Resumen}

Brasil es un importante productor de huevos y el Estado de São Paulo es el que más huevos produce en Brasil, siendo la región de Tupã el mayor productor del Estado con el 55\% de la producción y, en 2018 Bastos, la ciudad con el mayor producción, contribuyó con el 36\% del total de São Paulo. Así, con estos volúmenes de producción, es posible comprender la importancia de este sector productivo, que demanda innovaciones tecnológicas para garantizar la 
permanencia en el mercado y la eficiencia en la producción. La implementación de nuevas tecnologías para automatizar la producción de huevos no siempre trae solo resultados positivos, sino que requiere ajustes para evitar pérdidas durante el proceso de producción. En este contexto, se destaca el problema derivado de los sistemas automatizados de transporte de huevos, que pueden ocasionar pérdidas productivas y económicas a las granjas. Hay pérdidas en la línea de producción durante el transporte automatizado desde los almacenes hasta el proceso de envío final, donde las vibraciones e impactos provocan daños, así como los tiempos de exposición al sol. Considerando el escenario descrito, en este artículo el objetivo se basa en la prospección de la bibliografía que demuestra que ya existe tecnología desarrollada por pares para apoyar plenamente el futuro desarrollo tecnológico de un sistema electrónico autónomo para ser lanzado al sistema de transporte, recolectando datos sobre obstáculos, su posición y condiciones de exposición al mal tiempo, que se pueden comunicar con las computadoras del sistema de manejo de aviario y que pueden brindar información con el fin de mitigar las pérdidas productivas, aumentando la competitividad y seguridad alimentaria de las fincas.

Palabras clave: Revisión; Recopilador de datos; Software; Producción de huevos; Vibración.

\section{Introdução}

Segundo a Gessulli Agribusiness (2021) a produção brasileira de ovos totalizou 39 bilhões de unidades em 2016, um recorde que colocou o Brasil como sétimo maior produtor mundial. Quase tudo é consumido dentro do país e contribui para o aquecimento do mercado interno. Apesar de ter registrado um aumento de quase $40 \%$ desde 2010, há potencial para o consumo per capita aumentar muito no país. Atualmente, os brasileiros comem 190 unidades por ano. A média mundial é de 230, mas bate 300 ovos por pessoa em vários países, como China, Dinamarca e México.

Como pode ser observado na matéria publicada pelo Governo do Estado de São Paulo (2021a) na seção últimas notícias, São Paulo é o Estado que mais produz ovos do Brasil, concentrando 30,9\% da produção brasileira, uma produção capaz de alimentar mais de 60 milhões de pessoas por ano, considerando o consumo per capta de ovos no país, de 212 ovos por ano. Em 2018, o Brasil exportou 11,6 mil toneladas do alimento para todos os continentes do mundo, no valor de US\$17,1 milhões, segundo a Associação Brasileira de Proteína Animal (ABPA).

Nesta mesma matéria, há o destaque de que a Secretaria de Agricultura e Abastecimento do Estado de São Paulo afirma que a região de Tupã é a maior produtora de ovos no Estado com 55\% da produção, em 2018. Bastos, por sua vez, é o maior produtor de ovos no Estado, representando 36\% do total paulista. O Valor da Produção Agropecuária (VPA) de ovos de galinha é de $60,8 \%$ do VPA, da Regional de Tupã.

Ainda segundo a matéria publicada pelo Governo do Estado de São Paulo (2021a) no Estado foram produzidas 41 milhões de caixas de 30 dúzias de ovos no ano de 2018. Somente em Bastos foram produzidas 14,6 milhões de caixas de 30 dúzias de ovos em 2018 (ao redor de 5 bilhões de unidades). Além de que são produzidas por dia 60 mil caixas de 30 dúzias (21,6 milhões de unidades) sendo comercializado pelo preço médio de $\mathrm{R} \$ 68,99 / \mathrm{cx} .30 \mathrm{dz}$.

Segundo o levantamento de VPA, do Instituto de Economia Agrícola (IEA), a avicultura de postura no Escritório de Desenvolvimento Rural (EDR) de Tupã rendeu R \$ 1,5 bilhões naquele ano e no Estado foi de R \$ 2,8 bilhões e o município tem um plantel de 32 milhões de cabeças, sendo que 25 milhões de galinhas estão produzindo e 6 milhões são jovens. A alimentação delas é composta por 81 mil toneladas de ração por mês, 51 mil toneladas de milho por mês e 18 mil toneladas de farelo de soja mensais. A produção de ovos "in natura", líquidos, congelados, em pó e de aves de descarte em Bastos gera 4 mil empregos diretos e 8 mil indiretos na avicultura (Governo do Estado de São Paulo, 2021a).

Trata-se, portanto de importante cadeia produtiva da região e com grande potencial de crescimento.

Pizzolante et al. (2011) consideram que as inovações tecnológicas desempenham um papel muito importante como fator explicativo das estruturas e do comportamento das firmas. Dizem que na atividade da avicultura de postura as inovações tecnológicas que ocorreram recentemente podem ser consideradas radicais ou incrementais em sistemas de criação, manejo e genética das aves e, que a adoção de tecnologias pelo produtor tem tido como objetivo principal a redução de custos por meio de redução de tempo de processamento, uso de mão de obra e melhorias na logística interna e externa da firma. A sua 
permanência no mercado depende da sua eficiência na produção.

Segundo Silva et al. (2015), a avicultura brasileira vem incorporando sistemas automatizados, e o Brasil se tem mantido como um dos principais produtores mundiais de ovos de mesa. Os mesmos autores destacam que a qualidade do ovo é determinada por fatores externos e internos.

Quanto às instalações, conforme a pesquisa realizada por Pizzolante et al. (2011) os galpões se modernizaram, variando largura de 3 a $15 \mathrm{~m}$ e comprimento de 100 a $150 \mathrm{~m}$. Os autores destacam que nesses galpões são colocadas gaiolas com dois andares (sistema californiano) ou sistemas de baterias, com seis conjuntos de gaiolas sobrepostas umas nas outras, o que demanda grande esforço de produção.

Os mesmos autores reforçam dizendo que desta forma, a automação tornou-se presente em muitas granjas da região de Bastos e vão desde o fornecimento de ração e água até a coleta de ovos e, atualmente é possível que o produto final, o ovo, seja coletado nos galpões e chegue ao consumidor sem que haja contato manual, num processo totalmente automatizado. Os autores, ainda fazem destaque ao fato que, os ovos são coletados em esteiras que os conduzem para o depósito de ovos onde são levados diretamente para as máquinas de lavar, selecionar, classificar e embalar.

A implantação destas novas tecnologias para a automatização dos ambientes de produção de ovos, nem sempre trazem só resultados positivos, mas podem demandar ajustes e acessórios para que se evitem perdas durante o processo produtivo.

Considerando o disposto no trabalho de Nazareno (2012), hoje em dia observam-se perdas na linha de produção de ovos durante o transporte automatizado desde a produção desses ovos nos galpões até o processo final de embalagem e expedição, nos quais as vibrações e impactos de curta duração (impactos sofridos nos transportadores) causam danos (trincas/fissuras).

Do momento em que o ovo é posto até o local da sua comercialização, o principal objetivo é preservar sua qualidade original até que ele chegue ao consumidor, portanto, o transporte nas linhas automatizadas de produção de ovos dos aviários até o local de seleção e empacotamento deve ser realizado o mais breve possível a fim de reduzir as perdas iniciais da qualidade (Magalhães et al., 2012).

Considerando o cenário descrito anteriormente, neste artigo de revisão o gol está focado na busca de bases de fundamentação para um desenvolvimento tecnológico adicional, incremental, uma inovação tecnológica, na forma de um sistema eletrônico autônomo para ser lançado ao sistema de transporte desde a origem no local onde estão alojadas as aves até o ponto de chegada para a lavagem e embalagem dos ovos para expedição, passível de comunicação com computadores do sistema de gestão dos aviários que possa dar informações sobre localização e intensidade dos impactos de forma a mitigar as perdas produtivas oriundas dos sistemas de transporte entre os galpões e as unidades de processamento e expedição.

Esta pesquisa pretende embasar a hipótese de que é possível evitar a perda de qualidade dos ovos durante o transporte entre as unidades de processamento da granja a partir da análise dos dados armazenados digitalmente, do comportamento de exposição às variações bruscas de temperatura e umidade relativa do ar, vibrações e impactos detectados e registrados no sistema coletor proposto, por meio de uma interface computacional do tipo supervisório, a fim de se gerar uma ferramenta computacional com foco para o controle e mitigação do problema pela identificação dos defeitos localizados no trajeto das esteiras transportadoras e das utilidades dos entroncamentos entre galpões e unidade de expedição final dos ovos, auxiliando a manter o controle do trajeto do processo de maneira não intrusiva ao normal andamento do processo.

Isto irá ajudar as empresas a reduzir as perdas produtivas ocasionadas pelas vibrações do trajeto dos transportadores e também da exposição às bruscas variações de temperatura e, ampliar suas participações no mercado, ganhando assim maior qualidade, eficiência e consequentemente maior competitividade e lucratividade. 


\section{Metodologia}

Neste artigo, destaca-se a realização de uma revisão da bibliografia com aprofundamento no problema advindo dos sistemas de transporte dos ovos, que podem causar perdas produtivas e financeiras para as granjas.

O método adotado para a presente pesquisa, seguiu os passos de Pereira, A. S. et al (2018), com base na prospecção de documentos científicos e outros tipos de documentos nas bases nacionais e internacionais de artigos científicos. A técnica levou em conta as recomendações de que o observador, segundo afirmam Ludke \& Andre (2013), que o pesquisador precisa aprender a fazer registros descritivos, saber separar os detalhes relevantes dos triviais, aprendendo, assim a fazer anotações organizadas e utilizar rigorosos métodos para alcançar a validação de suas observações. Assim, seguindo estas considerações dos autores Köche (2011), Pereira, et al (2018) e Ludke e Andre (2013), adotou-se a Revisão Bibliográfica como base metodológica para a realização do presente estudo.

A delimitação geográfica desta pesquisa foi restrita aos produtores do município de Bastos / SP, Brasil, aproveitandose do fato de ser um importante centro e referência na produção de ovos. (Faria, 2013).

Foi realizada, então, uma prospecção da bibliografia em bases internacionais e nacionais de artigos científicos para o aprofundamento do entendimento sobre o estado da arte das pesquisas sobre este tipo de problema de vibrações e impactos em sistemas de transporte de ovos e as questões relativas à exposição às altas temperaturas e umidades variadas, e os eventuais riscos à produção, assim como as pesquisas já realizadas sobre o desenvolvimento de hardware e firmware para coletas de dados de sensores, desenvolvimento de softwares de supervisão e as técnicas utilizadas pelos pares e os métodos mais comumente aplicados para a obtenção de resultados em ambiente de produção de ovos. Foram prospectadas, preferencialmente, as bases Science Direct, Scielo e Scopus.

\section{Revisão da Bibliografia}

Segundo a pesquisa de Hunton (2005) e de Vilela et al. (2016) um fator determinante para a manutenção da qualidade do ovo é a integridade da casca considerada sua "embalagem" natural. A casca deve ser íntegra, sem deformações e trincas que comprometem seu conteúdo interno.

Defeitos nas cascas podem comprometer os ovos tanto para alimentação. Ainda não há domínio de todas as técnicas relacionadas ao manejo, necessárias para que os ovos atinjam os canais de mercado com a qualidade de casca desejada (Vieira el al, 2012).

Segundo Gherardi (2016), na avicultura de postura, perdas de enorme importância econômica para o avicultor estão relacionadas com a qualidade de casca dos ovos e aos índices de quebra. O aumento da qualidade da casca, portanto, terá um impacto econômico importante para a indústria.

Segundo Nazareno (2012) as perdas contabilizadas no transporte de ovos decorrentes da operação das linhas de produção caracterizam-se por ovos trincados e rachados e representam um grande desafio para as empresas integradoras. Além de resultarem em prejuízo, elas ainda apresentam dificuldades no que diz respeito ao desconhecimento dos locais fonte desta perda, bem como a busca de uma maneira de reduzi-las.

Podemos ver na pesquisa de Amaral et al. (2016) que a produção de ovos depende de um amplo conjunto de insumos, dentre os quais se se destacam as máquinas e equipamentos e a indústria de máquinas e equipamentos que fornecem todo o aparato necessário não só à criação das aves - como comedouros, bebedouros e coletores e transportadores de ovos -, mas também ao processamento e industrialização dos ovos.

Particularmente os transportadores de ovos que interligam as baterias de gaiolas verticais ou galpões de postura do tipo piramidal cage-free ou free-range às unidades de manejo para lavagem, classificação, empacotamento e expedição e, dependendo do comprimento total do sistema de transporte, tem seu sistema de configuração construtiva final normalmente 
composto ou por uma ou mais unidades de acionamento intermediárias.

As diferentes unidades de acionamento podem ser combinadas com outros modelos de transportadores (por exemplo, correias e sistemas elevadores verticais) e acomodam-se a variados tipos de curvas, com diferentes inclinações e elevadores com larguras eventualmente diferentes durante o trajeto, sendo que se completam com baias de orientação de fluxo dos ovos.

Estes sistemas transportadores são normalmente construídos em metal galvanizado e as correntes transportadoras de ovos são construídas com hastes de perfil arredondado em aço.

Entre as várias unidades de acionamento, tem-se acoplamentos mecânicos que introduzem impactos e vibrações e, os altos níveis de vibração podem aumentar a chance do aparecimento de trincas, microfissuras e rachaduras, além de ocasionar o agitamento dos elementos constituintes do ovo (Altuntas, 2008; Nazareno, 2012).

Ainda nesta linha de pesquisa, porém com foco no transporte com veículos, os autores Nazareno et al. (2013), realizaram uma pesquisa com o objetivo de caracterizar e avaliar a influência das estradas sobre os níveis de vibração e choques no transporte dos ovos férteis, avaliando dos níveis de vibração (m.s-2) e choques nas estradas de terra e asfalto foram registradas por meio de quatro acelerômetros. Os autores acabaram concluindo que os piores níveis de vibração e choque da carga de ovos ocorreram no sentido vertical (deslocamento da carga da frente para o fundo) em razão de ultrapassagens, frenagens bruscas e alta velocidade durante o transporte. Estes dados permitem agregar valor a esta pesquisa mediante os indicadores de danos causados pelos impactos.

Outra questão importante é a exposição dos ovos à irradiação solar e outras intempéries pelo intervalo de tempo que os ovos levam para sair do galpão de produção (postura) até chegarem ao local onde ocorre a lavagem, classificação, empacotamento e expedição, onde acabam sendo expostos a altas temperaturas e umidades relativas variadas, o que segundo Jones et al. (2002) pode afetar também a qualidade do produto final, pois, para a manutenção da alta qualidade dos ovos, os autores demonstraram que o tempo de trajeto a transcorrer e a temperatura ambiente e a umidade relativa são fatores importantes que devem ser controlados durante o período de transporte e armazenamento. Tudo isto é impactado ainda mais mediante a existência de fissuras e microfissuras nas cascas dos ovos.

Esta exposição, leva também a um problema sanitário, pois, sabe-se, por exemplo das pesquisas Oliveira \& Silva (2000), de que a contaminação dos ovos por salmonela se dá, inicialmente e na maioria das vezes, através da casca. Tempo e temperatura de armazenagem são fatores fundamentais para que as salmonelas passem da superfície da casca para as estruturas internas do ovo (Staldeman, 1986; Silva, 1995).

Desta forma, a aplicação da tecnologia de TI, segundo apresentado no trabalho de Souza et al. (2011), onde encontramos que atualmente no mercado equipamentos produzidos por grandes empresas do setor eletrônico adaptam os computadores do tipo PC para as mais diversas funções que vão desde a aquisição de dados até o controle de complexas linhas de produção na indústria. Os mesmos autores destacam que entre estes produtos estão as placas de aquisição de dados que, junto com um sortimento imenso de transdutores e sensores, permitem usar o PC para a medida e o registro das mais diferentes variáveis físicas, exatamente como proposto nesta pesquisa.

Segundo, ainda, os mesmos autores, um destes equipamentos é a placa didática de desenvolvimento Arduino, que é baseada num microcontrolador muito versátil, da família AVR do fabricante ATMEL, que potencializa suas funções para além de uma simples interface passiva de aquisição de dados, podendo operar sozinha no controle de vários dispositivos e tendo assim aplicações em instrumentação embarcada e robótica. Complementam, os autores, dizendo que todo o projeto eletrônico, incluindo a plataforma para o desenvolvimento dos programas de controle é de acesso público e gratuito podendo ser facilmente obtida em Arduino, (2021), com uma extensa comunidade internacional e na Web é possível encontrar uma farta documentação sobre aplicações e acessórios.

Outros trabalhos utilizando esta plataforma de hardware, em diferentes aplicações e áreas de conhecimento já foram 
realizados por Kamogawa e Miranda (2013); Cavalcante et al. (2011).

Particularmente os autores Patel e Patel (2017), pesquisaram uma plataforma Arduino com a utilização de um sensor de vibração baseado em um acelerômetro de três eixos, a qual chamaram de unidade de deteç̧ão inteligente para medição de vibração e monitoramento de condições de máquinas. Os autores acabaram por concluir que por este tipo de sistema de módulo de deteç̧ão de vibração, pode-se ter certeza de que a fidelidade da medição de vibração é mantida e, portanto, é possível tomar decisões confiáveis sobre práticas de manutenção preventiva para várias máquinas nas indústrias. Destacam ainda que consequentemente ao uso deste dispositivo, haverá economia de custos; tempo e recursos humanos também serão economizados. Portanto, complementam afirmando que, o módulo de detecção inteligente garante robustez e fidelidade da medição de vibração.

Neste campo do conhecimento desponta também a agricultura de precisão, que pode ser definida como no trabalho de Srbinovska et al. (2015), como sendo a arte e ciência que usa tecnologia avançada para melhorar a produção agrícola. Os autores apontam para tecnologias compostas por sensores e computadores que são as maiores causadoras do desenvolvimento da agricultura de precisão. Destacam ainda o fato que, os recentes avanços nas comunicações e eletrônica permitiram o desenvolvimento e produção de baixo custo e baixo consumo de energia, de sensores multifuncionais, que são pequenos em tamanho e podem se comunicar de maneira inteligente e barata, e podem ser implantados em grandes números, proporcionando enormes oportunidades para o monitoramento e controle de ambientes (Vieira et al., 2003).

\section{Resultados e Discussão}

Segundo os resultados obtidos na prospecção das bibliografias disponíveis, inicialmente observa-se a grande relevância do tema em questão para o setor produtivo.

Este setor produtivo da economia tem um potencial muito grande como destacou o artigo do pelo Governo do Estado de São Paulo (2021b) e que representa para o Brasil anualmente um volume de exportação de 11,6 mil toneladas do alimento para todos os continentes do mundo, no valor de US\$17,1 milhões, segundo a Associação Brasileira de Proteína Animal (ABPA).

Como é possível, também, observar da pesquisa apresentada por Gherardi (2016), que destaca a importância da qualidade das cascas dos ovos e dos trabalhos de Nazareno (2012) e de Nazareno et al. (2013) que abordam as perdas produtivas devido ao processo na linha de produção de ovos durante o transporte automatizado e nos quais as vibrações e impactos de curta duração (impactos sofridos nos transportadores e veículos de transporte) causam danos (trincas/fissuras) pelas quais podem ingressar patógenos importantes o que afetam sobremaneira a segurança alimentar.

Deve-se destacar também que a região em que a pesquisa futura com implantação de hardware e softwares de monitoramento será desenvolvida e que pretende trazer a inovação tecnológica é a região de Tupã, na Alta Paulista, no oeste de São Paulo, vizinha à cidade de Bastos, na qual se pretende instalar o equipamento proposto, é responsável segundo a matéria publicada pelo Governo do Estado de São Paulo (2021b) da produção 41 milhões de caixas de 30 dúzias de ovos só no ano de 2018, o que financeiramente remonta a uma quantia que não deve ser desconsiderada.

\section{Conclusão}

Desta forma, foi possível concluir com a revisão das bibliografias disponíveis sobre o tema de vibração em transporte de ovos apresenta de maneira incisiva os impactos negativos à segurança alimentar e à lucratividade das granjas, o que deve ser combatido por meio de automação com sensores e dispositivos eletrônicos de coleta de dados e computadores para localizar as falhas no processo mitigando estas fontes de fissuras e microfissuras à camada protetora, ou casca, dos ovos. Observou-se também a possibilidade de se obter, por meio da implantação destes dispositivos um impacto positivo que pode ser gerado com 
a inovação deste produto proposto, com a redução das perdas produtivas anteriormente destacadas, só nesta região, e posterior possibilidade de expansão para todas as granjas do país.

Como sugestão para trabalhos futuros, já que há disponível a tecnologia desenvolvida pelos pares para dar suporte integral ao desenvolvimento tecnológico de um sistema eletrônico autônomo para ser lançado ao sistema de transporte, coletando dados dos obstáculos, sua posição e condições de exposição às intempéries, passível de comunicação com computadores do sistema de gestão dos aviários, considera-se importante, o desenvolvimento de um protótipo funcional do sistema coletor proposto, por meio de uma interface computacional do tipo supervisório, a fim de se gerar uma ferramenta computacional robusta com foco para o controle e mitigação do problema pela identificação dos defeitos localizados no trajeto das esteiras transportadoras e das utilidades dos entroncamentos entre galpões e unidade de expedição final dos ovos, auxiliando a manter o controle do trajeto de maneira não intrusiva ao normal andamento do processo.

\section{Agradecimentos}

O autor agradece ao CNPq Processo DT-II n $313339 / 2019-8$ pelo suporte às pesquisas.

\section{Referências}

Altuntas, E. \& Şekeroğlu, A., (2008). Effect of egg shape index on mechanical properties of chicken eggs; Original Research Article Journal of Food Engineering, 85(4), 606-612.

Amaral, G., Guimarães, D., Nascimento, J. C.\& Custodio, S. (2016). Avicultura de postura: estrutura da cadeia produtiva, panorama do setor no Brasil e no mundo e o apoio do BNDES. Agroindústria, BNDES Setorial 43(1), 167-207.

Arduino. (2021). Download the Arduino Software. Website Arduino.cc.

Cavalcante, M. A., Tavolaro, C. R. C., \& Molisani, E. (2011). Física com Arduino para iniciantes. Rev. Bras. Ensino Fís., 33(4). http://www.scielo.br/scielo.php?script=sci_arttext\&pid=S1806-11172011000400018\&lng=pt\&nrm=iso

Faria, J. M. (2013). Dinâmica estrutural do setor produtivo de ovos: uma análise a partir das empresas líderes brasileiras. Rio Grande do Sul: UFRGS. 113p. Dissertação (Mestrado) - Programa de Pós-Graduação em Agronegócios, Universidade Federal do Rio Grande do Sul, Porto Alegre.

Gessulli agribusiness. (2021). Brasil é sétimo maior produtor mundial de ovos. Revista digital Avicultura Industrial.com.br. https://www.aviculturaindustrial.com.br/imprensa/brasil-e-setimo-maior-produtor-mundial-de-ovos/20171113-144114-a215

Gherardi, S. R. M. \& Vieira, R. P. (2016). Fatores que afetam a qualidade da casca do ovo: revisão de literatura. Nutritime Revista Eletrônica, on-line, Viçosa, 13(3), 8172-8181. ISSN: 1983-9006

Governo do Estado de São Paulo. (2021a). Com 5 bilhões de ovos por ano, Bastos é maior produtora de SP. Do Portal do Governo. http://www.saopaulo.sp.gov.br/spnoticias/com-5-bilhoes-de-ovos-por-ano-bastos-e-maior-produtor-do-estado/

Governo do Estado de São Paulo. (2021b). IEA divulga o Valor da Produção Agropecuária Paulista Regional: Resultado Final 2018. Secretaria de Agricultura e Abastecimento - Instituto de Economia Agrícola (IEA), 2019. http://www.iea.sp.gov.br/out/TerTexto.php?codTexto=14613

Hunton P. (2005). Research on eggshell structure and quality: An historical overview. Revista Brasileira de Ciência Avícola. 7(2), 67-71.

Jones, D. R., Tharrington, J. B., Curtis, P. A., Anderson, K. E., Keener, K. M. \& Jones, F. T. (2002). Effects of cryogenic cooling of shell eggs on egg quality. Poultry Science, 81(5), 727-733.

Kamogawa, M. Y. \& Miranda, J. C. (2013). Uso de hardware de código fonte aberto "Arduino" para acionamento de dispositivo solenoide em sistemas de análises em fluxo. Química Nova, São Paulo, 36(8), 1232-1235.

Köche, J. C. (2011). Fundamentos de metodologia científica: teoria da ciência e iniciação à pesquisa. Vozes.

Ludke, M. \& Andre, M. E. D. A. (2013). Pesquisas em educação: uma abordagem Qualitativa. E.P.U. F.

Magalhães, A. P. C, Curvello, F. A., Morenz, M. J., Calixto, L. F. \& Rezende, S. R. F. (2012). Qualidade de ovos comerciais de acordo com a integridade da casca, tipo de embalagem e tempo de armazenamento. Revista de Ciência da Vida, 32(1), 51-62.

Nazareno, A. C., Iran Silva, J. O., Vieira, A. M. C. Vieira, F. M. C. \& Miranda, K. O. S. (2013). Níveis de vibração e choques em diferentes estradas durante o transporte de ovos férteis. Revista Brasileira de Engenharia Agrícola e Ambiental. 17(8), 900-905.

Nazareno, A. C. (2012). Ambiência pré-porteira; avaliação das condições bioclimáticas e das operações pré-eclosao na qualidade de pintos de corte. Tese (Doutorado em física do Ambiente Agrícola) - Escola Superior de Agricultura "Luiz de Queiros”, Universidade de São Paulo, 85-117.

Oliveira, A. B. \& Chiari, R. (2015). Fundamentos em gerenciamento de projetos baseado no PMBOK 5A Edição. Communit, 2015. https://amauroboliveira.files.wordpress.com/2015/11/fundamentos-em-gerenciamento-de-projetos.pdf 
Oliveira, D. D., \& SILVA, E.N. (2000). Salmonela em ovos comerciais: ocorrência, condições de armazenamento e desinfecção da casca. Arquivo Brasileiro de Medicina Veterinária e Zootecnia, 52(6), 655- 661.

Patel, V. K. \& Patel, M. N. (2017). Development of Smart Sensing Unit for Vibration Measurement by Embedding Accelerometer with the Arduino Microcontroller. International Journal of Instrumentation Science, 6(1), 1-7.

Pereira, A. S., Shitsuka, D. M., Parreira, F. J. \& Shitsuka, R. (2018). Metodologia da pesquisa científica. UFSM.

Pizzolante, C.C., Saldanha, E.S.P.B., Moraes, J.E. \& Kakimoto, S.K. (2011) A trajetória tecnológica na avicultura de postura. APTA - Pesquisa \& Tecnologia, 8(2), 1-6. http://aptaregional.sp.gov.br/acesse-os-artigos-pesquisa-e-tecnologia/edicao-2011/2011-julho-dezembro/1010-a-trajetoria-tecnologica-na-aviculturade-postura/file.html

Silva, E.N. (1995). Salmonella enteritidis em aves e saúde pública. Hig. Alimentar, 9(1), 7-13.

Silva, R.C., Nascimento, J. W. B., Oliveira, D. L. \& Furtado, D. A. (2015). Termohigrometria no transporte e na qualidade de ovos destinados ao consumo humano. Revista Brasileira de Engenharia Agrícola e Ambiental, UAEA/UFCG 19(7), 668-673.

Souza, A. R., Paixão, A. C., Uzêda, D. D.; Dias, M. A., Duarte, S. \& Amorim, H. S. (2011). A placa Arduino: uma opção de baixo custo para experiências de física assistidas pelo PC. Rev. Bras. Ensino Fís., São Paulo, 33(1), 1702-1-1702-5.

Srbinovska, M., Gavrovski, C., Dimcev, V., Krkoleva, A. \& Borozan, V. (2015). Environmental parameters monitoring in precision agriculture using wireless sensor networks. Journal of Cleaner Production, 88(1), 297-307.

Vieira, D. V. G., Barreto S. L. T., Valeriano M. H., Jesus L. F. D., Silva L. F. F., Mencalha R., Barbosa K. S., Mendes. R. K. V., Cassuce, M. R. \& Melo, T. S. (2012). Exigências e cálcio e de fósforo disponível para codornas japonesas de 26 a 38 semanas de idade, Revista Brasileira de Saúde e Produção Animal, 13(1), 204-13.

Vieira, M. A. M., Coelho. JR., C. N., Silva Junior, D. C. \& Mata, J. M. (2003). Survey on Wireless Sensor Network Devices. Emerging Technologies and Factory Automation. Proceedings. ETFA '03. IEEE Conference, 1(1), 537-544.

Vilela, D. R., Carvalho, L. S., Fagundes, N. S. \& Fernandes, E. A. (2016). Qualidade interna e externa de ovos de poedeiras comerciais com cascas normal e vítrea. Ciência Animal Brasileira, 17(4), 509-518. 